\title{
Spatio-Temporal Graph Routing for Skeleton-Based Action Recognition
}

\author{
Bin $\mathrm{Li},{ }^{1} \mathrm{Xi} \mathbf{L i},{ }^{2 *}$ Zhongfei Zhang, ${ }^{1}$ Fei Wu ${ }^{2}$ \\ ${ }^{1}$ College of Information Science \& Electronic Engineering, Zhejiang University, Hangzhou, China \\ ${ }^{2}$ College of Computer Science and Technology, Zhejiang University, Hangzhou, China \\ \{bin_li, xilizju, zhongfei\}@zju.edu.cnwufei@cs.zju.edu.cn
}

\begin{abstract}
With the representation effectiveness, skeleton-based human action recognition has received considerable research attention, and has a wide range of real applications. In this area, many existing methods typically rely on fixed physicalconnectivity skeleton structure for recognition, which is incapable of well capturing the intrinsic high-order correlations among skeleton joints. In this paper, we propose a novel spatio-temporal graph routing (STGR) scheme for skeletonbased action recognition, which adaptively learns the intrinsic high-order connectivity relationships for physicallyapart skeleton joints. Specifically, the scheme is composed of two components: spatial graph router (SGR) and temporal graph router (TGR). The SGR aims to discover the connectivity relationships among the joints based on sub-group clustering along the spatial dimension, while the TGR explores the structural information by measuring the correlation degrees between temporal joint node trajectories. The proposed scheme is naturally and seamlessly incorporated into the framework of graph convolutional networks (GCNs) to produce a set of skeleton-joint-connectivity graphs, which are further fed into the classification networks. Moreover, an insightful analysis on receptive field of graph node is provided to explain the necessity of our method. Experimental results on two benchmark datasets (NTU-RGB+D and Kinetics) demonstrate the effectiveness against the state-of-the-art.
\end{abstract}

\section{Introduction}

As a challenging problem in computer vision, skeletonbased human action recogntion takes $3 \mathrm{~d}$ human body coordinates as input and outputs action class, which attracts increasing attention recently (Wang et al. 2018b). Typically, human body skeletons characterize the geometric body configuration as rigid body, and their dynamics capture motion patterns in a continuous way. This dynamic geometric structure expresses relation among the joints not only spatially but also temporally. By this means, graph representation is the natural way to express the intrinsic human structure. Therefore, it is crucial to automatically represent joints on the given graph. Recent success of Spatial Temporal Graph Convolution Networks (ST-GCN) (Yan, Xiong, and Lin 2018) has justified the effectiveness by a graph

\footnotetext{
${ }^{*}$ Corresponding author: $\mathrm{Xi} \mathrm{Li}$

Copyright (C) 2019, Association for the Advancement of Artificial Intelligence (www.aaai.org). All rights reserved.
}

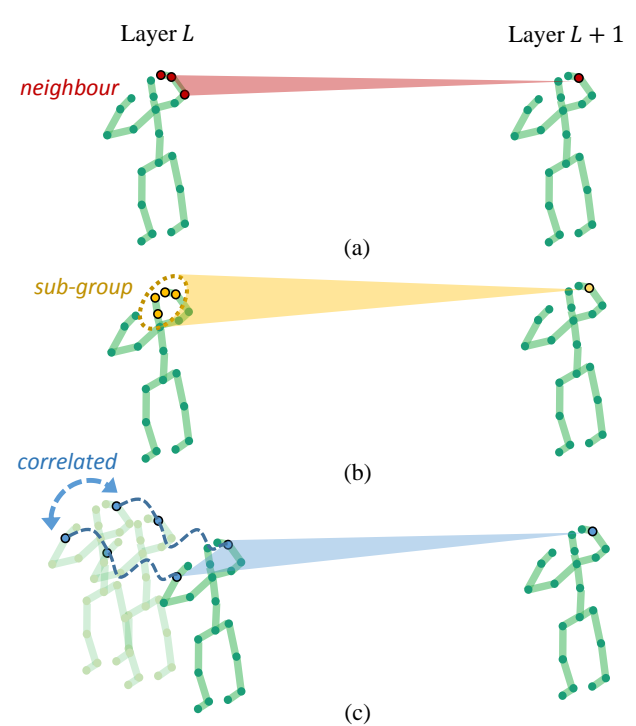

Figure 1: Illustraion of three routing ways: (a) fixed routing by physical connections; (b) spatial routing by considering local clustering; (c) temporal routing by modeling the correlation degrees of node trajectories.

aggregation scheme with physical human skeleton, against the existing literatures such as pseduo images (Wang et al. 2018a; Xie et al. 2018), variants of LSTM (Shahroudy et al. 2016; Song et al. 2017; Liu et al. 2017).

In general, the graph-based method applies a fixed human skeleton to graph convolution operation and iteratively aggregates the hidden feature with neighbourhood features. However, it is challenging to capture changeable human structure in complex scene. This brings three-fold problems for further improvement: 1) The skeleton itself is changeable and depends on specific dataset, e.g., 25 joints in NTURGB+D (Shahroudy et al. 2016) while 18 joints in Kinetics (Kay et al. 2017), resulting in confusion on real human skeleton; 2) The joint connections are highly unbalanced. While torso joints become over-smoothing, limb joints may still be under-smoothing, which causes extreme difficulty on feature sharing for two limb joints; 3 ) A global graph structure is applied to each sample, raising the question "one size 
fits all", which is possibly sub-optimal. With a fixed graph, dataflow is restricted in predefined entries, which greatly decreases the flexibility of the model. We term this as "static routing" by analogy with Computer Networking.

In contrast, we pay much attention to seeking more flexible connection scheme, which adaptively learns the intrinsic high-order connectivity among skeleton joints for specific sample, referring to "dynamic routing". In real world scenarios, the dynamic skeleton itself embeds rich information that implicitly shows strong connection between two physically apart joints, e.g., two hand joints in action class "clapping". Therefore, we formulate this dynamic routing problem as a graph topology learning problem that automatically select the most informative connections for all joints. We show that dynamic routing scheme and static routing scheme are equally important in the task.

Motivated by this observation, we formulate this problem as a joint learning problem. We first learn dynamic graph topology via position and motion of the skeleton and then apply them as a prior to the GCN recognition framework. In particular, we propose a novel Spatio-Temporal Graph Routing (STGR) scheme to model the semantic connections among the joints in a disentangled way. Rather than using fixed human skeleton, two sub-networks are responsible to capture both spatial and temporal dependancies between each two nodes, serving as routers for all nodes. As shown in Figure 1, a spatial graph router (SGR) discovers the connectivity relationships among the joints based on sub-group clustering along the spatial dimension. A temporal graph router (TGR) explores the structural information by measuring the correlation degrees between temporal joint node trajectories. The spatio-temporal skeleton-joint-connectivity graphs are then fed into ST-GCN in multiple routing ways.

To explain the necessity, we further introduce "receptive field on graph" by analogy with the same term in CNNs. Receptive field on graph refers to coverage range that a node can draw information from. By introducing this concept, we show that fixed human skeleton would lead to highly unbalanced problem, which could be solved by our work.

Our contribution can be summarized as follows:

- We propose a novel spatio-temporal graph routing scheme, which is used to exploit intrinsic high-order relationship among skeleton joints. The module is jointly learned with classification network and better matches the action recognition task.

- We present receptive field on graph nodes to prove that the bottleneck of previous model is unbalanced receptive field for different joints, which shows effectiveness of our spatio-temporal graph routing scheme.

\section{Related Works}

Skeleton-based Action Recognition. Traditional approaches for skeleton-based action recognition mainly focus on hand-crafted features to capture the dynamics of joint motion, such as covariance matrix of the trajectories(Hussein et al. 2013), lie groups (Vemulapalli, Arrate, and Chellappa 2014).
With the success of the deep learning, many CNN-based methods are proposed in an end-to-end manner.To better ultilize existed powerful structures, many efforts have been made to transform raw skeleton into pseduo image in multiple ways, including skepxels(Liu, Akhtar, and Mian 2017), temporal-then-spatial recalibration scheme(Xie et al. 2018), and jointwise co-occurence(Li et al. 2018a).

Recurrent Neural Networks, on the other hand, effectively models temporal dependency. LSTMs and GRUs are proposed to learn temporal context of sequences(Shahroudy et al. 2016; Li et al. 2017). To better handle complex spatiotemporal variation factors, attention mechanism is proposed to ensure requirement of robustness, such as key frame selection (Song et al. 2017) and global informative joints mining(Liu et al. 2017).

Graph Neural Networks. Graph neural networks (GCNs) can be roughly categorized into two streams: 1) spectral domain, which is based on the Graph Fourier Transform (GFT) (Shuman et al. 2013), performs transformation on graph basis(Bruna et al. 2014). By a parameterization of Klocalized convolutions, computationally efficient and localized filtering has been recently achieved(Defferrard, Bresson, and Vandergheynst 2016). To alleviate expensive cost of computing eigenvalues of Laplacian matrix, Chebyshev polynomials are introduced as a truncated expansion (Hammond, Vandergheynst, and Gribonval 2011). 2) spatial domain, on the other hand, learns to aggregate each node's neighbourhood as its new hidden representation iteratively. A first-order approximation is proposed(Kipf and Welling 2017), which succeeds in semi-supervised classification. Meanwhile, some literature(Niepert, Ahmed, and Kutzkov 2016) explores to greedly converts graph into sequence and make use of 1D convolution networks.

Recently, a few works attempt to reveal the mechanism of GCNs with either metric learning (Li et al. 2018b) or jumping knowledge networks (Xu et al. 2018). However, current methods are mainly focused on semi-supervised classification problem. In this work, we first conduct analysis on skeleton-based action recognition with the help of receptive field on graph.

\section{Methods}

In this section, we first formulate our problem and then introduce our Spatio-Temporal Graph Routing (STGR) scheme by describing two sub-networks-SGR and TGR respectively. Later we describe the overall architecture and optimization. At last, we discuss receptive field on graph to further verify the necessity of STGR.

\section{Problem Formulation}

A 3D human skeleton is denoted as $X=\left\{x_{n}^{t}\right\} \in$ $\mathbb{R}^{C_{\text {in }} \times T \times N}$ with $T$ frames and $N$ joints. Each individual is represented as a $x y z$-coordinate feature vector for $n$-th joint at $t$-th time step and hence $C_{\text {in }}=3$. For further convenience, we describe single frame skeleton as $X^{t} \in \mathbb{R}^{C_{\text {in }} \times N}$ for $t$-th frame and node trajectory as $X_{n} \in \mathbb{R}^{C_{\text {in }} \times T}$ for $n$-th joint.

Let $A \in \mathbb{R}^{N \times N}$ be the simple adjacency matrix whose entry $A_{i j}$ denotes whether joint $i$ and joint $j$ are connected. 


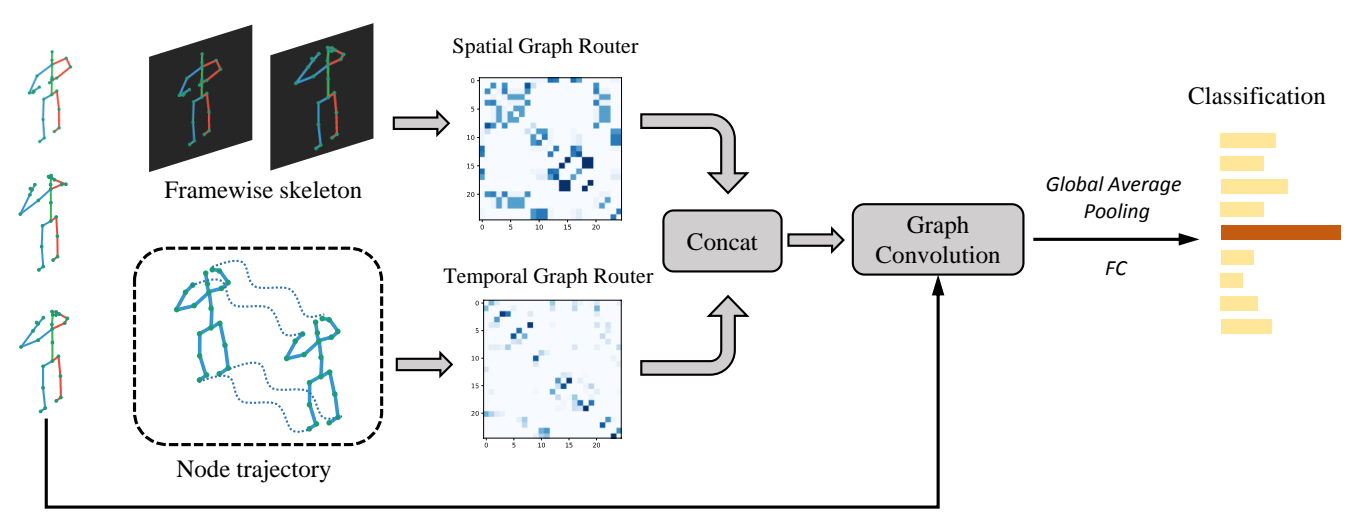

Figure 2: Overview of spatio-temporal graph router. The input 3d-skeleton sequence is first transformed as framewise skeleton and node trajectories respectively. Then Spatial Graph Router (SGR) and Temporal Graph Router (TGR) produce new skeleton-joint-connectivity graphs respectively. ST-GCN receives this graphs and outputs action class.

$D$ is the corresponding degree matrix. The default graph is denoted by:

$$
\mathcal{G}^{\text {default }}=\tilde{D}^{-\frac{1}{2}} \tilde{A} \tilde{D}^{-\frac{1}{2}}
$$

where $\tilde{A}=A+I$ is the generalized adjacency matrix including node itself. $\tilde{D}$ is the corresponding degree matrix of $\tilde{A}$. Thus, $\mathcal{G}^{\text {default }}$ is the diagonal normalized matrix of $\tilde{A}$.

As discussed above, the fixed human skeleton is insufficient to model the changeable human structure in complex scenes. Our goal is to learn the mapping from the raw skeleton to the graph topology representation: $X \rightarrow \mathcal{G}$ in multiple views such as pose and motion. Therefore we have:

$$
\left\{\mathcal{G}^{\text {spat }}, \mathcal{G}^{\text {temp }}\right\}=f_{\text {STGR }}\left(X ; \theta^{\text {spat }}, \theta^{\text {temp }}\right)
$$

where $\mathcal{G}^{\text {spat }}$ and $\mathcal{G}^{\text {temp }}$ are spatial and temporal graph topology representation. $\theta^{\text {spat }}$ and $\theta^{\text {temp }}$ represent corresponding parameters. $\mathcal{G}^{\text {spat }}$ and $\mathcal{G}^{\text {temp }}$ will concatenate with default graph $\mathcal{G}^{\text {default }}$ to form a graph set $\mathcal{S}=\left\{\mathcal{G}^{\text {default }}, \mathcal{G}^{\text {spat }}, \mathcal{G}^{\text {temp }}\right\}$. In the following parts, we will provide a detailed description on two sub-networks.

\section{Spatial Graph Router Sub-network}

In real world scenarios, joints usually gather in a group to express a specific action. In other words, the position of each joint and the distance between pairwise joints encode the intensity of the relation, which is crucial to guide information flow.

Spatial Graph Pool. In order to extract spatially connected graph, we first use a non-parametric graph cut clustering method (Shi and Malik 2000) for each frame skeleton $X^{t} \in \mathbb{R}^{C_{\text {in }} \times N}$, forming $K$ sub-groups. As for each subgroup, we treat it as a completely connected graph, which means each two nodes are connected within the same subgroup. In this way, we define a spatially connected graph for each frame $t$ and gather all these graphs to form the "Spatial Graph Pool":

$$
\mathcal{G}=\left\{\mathcal{G}^{1}, \ldots, \mathcal{G}^{T}\right\}
$$

where for each single $\mathcal{G}^{t}$ :

$$
\mathcal{G}_{i j}^{t}= \begin{cases}1, & \text { if } i \text { and } j \text { in the same sub-group } \\ 0, & \text { otherwise }\end{cases}
$$

Squeeze-and-Excitation Attention. Since we have already obtained a series of spatially connected graphs, our goal is to select the most informative one as representative. To this end, a frame attention scheme for jointly learning framewise importance is proposed for graph fusion.

As shown in Figure 3, we model the frame attention in a Squeeze-and-Excitation way (Hu, Shen, and Sun 2018). A large $7 \times 7$ convolution is first applied to aggregate local feature. Squeeze operation is then conducted via a global average pooling layer to obtain the intermediate feature:

$$
m_{t}^{\text {in }}=\frac{1}{N \times N} \sum_{i=1}^{N} \sum_{j=1}^{N} f_{\text {Conv }}\left(\mathcal{G}^{t}\right)_{i j}
$$

where $m^{\text {in }}=\left(m_{1}^{\text {in }}, \ldots, m_{T}^{\text {in }}\right)$ denotes a collection of intermediate features in temporal space. Since $m^{\text {in }}$ contains the complete information for the whole graph, the Excitation operation can model the internal dependancy across frames.

$$
\mu=\operatorname{Sigmoid}\left(W_{2} \cdot \sigma\left(W_{1} \cdot m^{\text {in }}\right)\right)
$$

where $\mu=\left(\mu_{1}, \ldots, \mu_{T}\right)$ denotes importance score for each frame. $W_{1} \in \mathbb{R}^{\frac{T}{r} \times T}$ and $W_{2} \in \mathbb{R}^{T \times \frac{T}{r}}$ are $1 \times 1$ transformation matrix. $r$ is the dimension reduction parameter and $\sigma$ is ReLU activation function. This dimensionality reduction scheme is mainly used for exploiting the relations for temporal dimension. We make a weighted fusion with each of its frame importance $\mu_{t}$ to form the $\mathcal{G}^{\text {spat: }}$

$$
\mathcal{G}^{\text {spat }}=\frac{1}{T} \sum_{t=1}^{T} \mu_{t} \cdot \mathcal{G}^{t}
$$




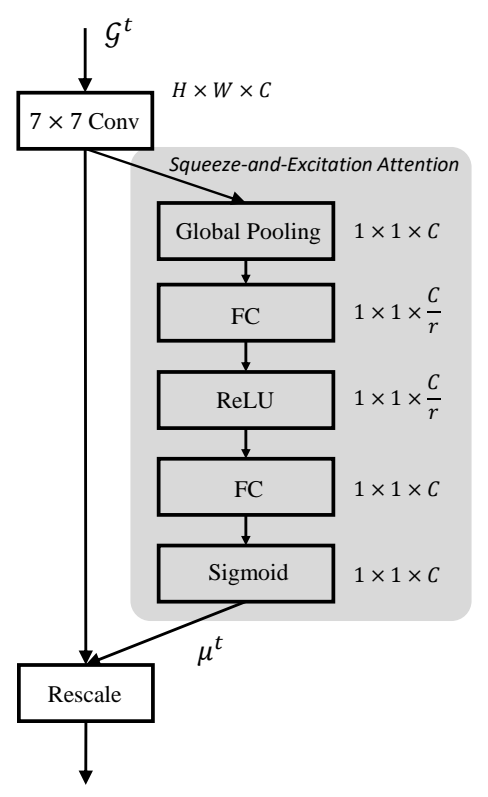

Figure 3: Network structure for Spatial Graph Router (SGR).

\section{Temporal Graph Router Sub-network}

Different from SGR sub-network, TGR sub-network considers the spatio-temporal graph in a global way. Based on a simple observation, joints with high correlation degrees usually implies close relation. For example, in class "walking", hands and feet are highly correlated(swing in opposite directions), indicating the discriminative relation. Inspired by this idea, TGR first encodes each node trajectory with a LSTM encoder, then models relation for each pair nodes in a selfattentive way.

LSTM encoder. As discussed above, TGR first rearranges the input sequence as $N$ independant node trajectories $X_{n}, n=1, \ldots, N$, each of which is regarded as $X_{n} \in$ $\mathbb{R}^{C_{\text {in }} \times T}$. As shown in Figure 4, a LSTM unit first encodes each input node trajectory and outputs the hidden state at the last time step:

$$
h_{n, t}=\psi\left(X_{n}, h_{n, t-1}\right)
$$

where $\psi$ denotes LSTM module. $h_{n, t}$ is the output at the last time steps. $h_{n, t}$ is then taken as input of the relation modeling network to capture the interaction between two nodes. For clarity, we denote $h_{n, t}$ as $v_{n}$ for each node.

Relation modeling. We model the pairwise node relation in the encoded feature space. Similar to rencent work (Wang et al. 2018c), we measure the relation by normalized dot product. Particularly, given each trajectory's encoded feature $v=\left[v_{1}, \ldots, v_{N}\right]$, The pairwise similarity is proposed as:

$$
D\left(v_{i}, v_{j}\right)=\theta\left(v_{i}\right)^{\top} \cdot \varphi\left(v_{j}\right)
$$

where $\theta$ and $\varphi$ are two $1 \times 1$ transformation operations. By performing a dot-product, we examine two nodes by their cosine distance. After computing each pairwise distance, we

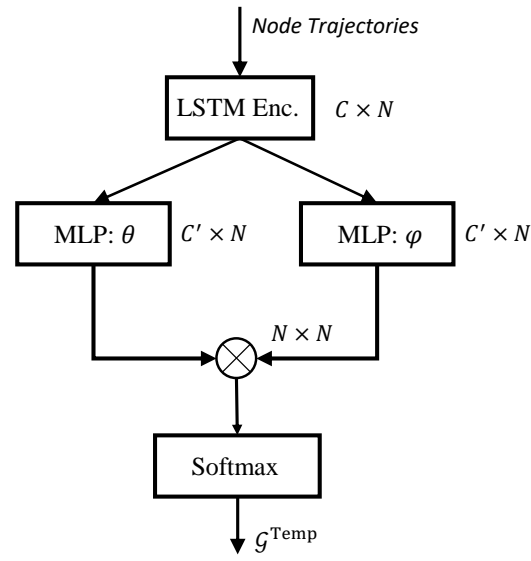

Figure 4: Network structure for Temporal Graph Router (TGR).

further apply a softmax operation in each row, ensuring sum of all entries of a single node will be set to 1 .

$$
\mathcal{G}_{i j}^{\text {temp }}=\frac{\exp D\left(v_{i}, v_{j}\right)}{\sum_{k=1}^{N} \exp D\left(v_{k}, v_{j}\right)}
$$

\section{Network Architecture and Optimization}

In this section, we introduce the overall network architecture. Our model is constructed by STGR and ST-GCN. In particular, STGR is responsible to explore intrinsic connectivity relationships for semantically related joints in both spatial and temporal domains. ST-GCN takes both 3D skeleton and the graph as input and output action class. In particular, ST-GCN stacks multiple "GCN-TCN" units(Yan, Xiong, and Lin 2018) for representation learning, of which each "GCN-TCN" unit is seen as one layer. Each GCN unit performs graph convolution operation with default graph $\mathcal{G}^{\text {default }}$ and learned graph $\mathcal{G}^{\text {spat }}$ and $\mathcal{G}^{\text {temp }}$ in spatial dimension while TCN unit is applied in temporal dimension to get high-level feature maps.

To make it clear, suppose hidden feature of specific node $n$ in $l$-th layer is denoted by $h_{v}^{l} \in \mathbb{R}^{d_{l}}$. For consistency, we assume $h^{0}=X$ and $d_{0}=C_{\text {in }}$. Vanilla ST-GCN can then be interpreted as:

$$
h_{v}^{l+1}=\sigma\left(\left(M \otimes \mathcal{G}^{\text {default }}\right) h_{v}^{l} w^{l}\right)+h_{v}^{l}
$$

where $M$ represents a learnable mask to further enlarge the model's expressive power. $\otimes$ is element-wise product and $w^{l}$ denotes a regular convolution operation right after graph convolution. Along with our STGR, we produce a series of spatial and temporal graph $\mathcal{G}_{i}^{\text {spat }}, \mathcal{G}_{i}^{\text {temp }}, i=1, \ldots, L$ for each layer respectively. We do not share weights of each unit of STGR since the model is lightweight.

Therefore, the spatial and temporal graph is embedded into each "GCN-TCN" unit. Joints can aggregate features from not only fixed skeleton but also these learned semantic connections: 


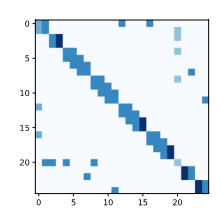

Graph Representation
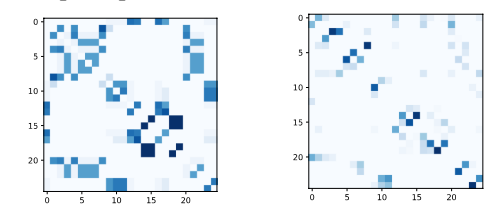

Visualization of connections

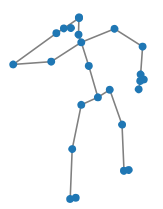

(a)

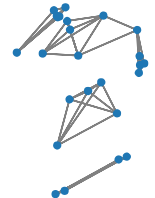

(b)

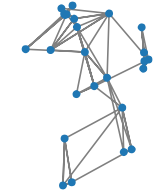

(c)
Figure 5: Comparison of 3 types of connections. (a) physical connection; (b) learned spatial connection with SGR; (c) learned temporal connection with TGR. Above are matrix representaions of graphs. Below are the corresponding visualizations of joint connections. For better view, the connections are binarized with threshold 0.05 in visualizations.

$$
h_{v}^{l+1}=\sigma\left(\sum_{\mathcal{G} \in \mathcal{S}}\left(M_{\mathcal{G}} \otimes \mathcal{G}\right) h_{v}^{l} w_{\mathcal{G}}^{l}\right)+h_{v}^{l}
$$

where $\mathcal{S}=\left\{\mathcal{G}^{\text {default }}, \mathcal{G}^{\text {spat }}, \mathcal{G}^{\text {temp }}\right\} . M_{\mathcal{G}}$ and $w_{\mathcal{G}}^{l}$ are the corresponding mask and convolution for the specific graph. We stack multiple GCN-TCN units and then apply global average pooling and full connected layer to obtain the action score $\hat{y}$ :

$$
\hat{y}=f_{\text {ST-GCN }}\left(X ; \mathcal{G}_{1}^{\text {spat }}, \mathcal{G}_{1}^{\text {temp }}, \ldots \mathcal{G}_{L}^{\text {spat }}, \mathcal{G}_{L}^{\text {temp }}\right)
$$

We employ standard cross-entropy loss for classification. As for the two sub-networks, to ensure the graph sparsity, the $L 1$ loss is employed:

$$
\begin{aligned}
& \mathcal{L}_{\text {cls }}=-\sum_{i=1}^{M} y_{c} \log \left(\hat{y}_{i}\right), \\
& \mathcal{L}_{\text {sparse }}=\sum_{i=1}^{L}\left\|\mathcal{G}_{i}^{\text {spat }}\right\|_{1}+\left\|\mathcal{G}_{i}^{\text {temp }}\right\|_{1}, \\
& \mathcal{L}=\mathcal{L}_{\text {cls }}+\lambda \mathcal{L}_{\text {sparse }}+\|\Theta\|_{2}
\end{aligned}
$$

where $M$ is the overall the number of action classes, $y_{c}$ represents the ground truth label. $\Theta$ is the overall parameters for both ST-GCN and STGR. $\lambda$ is used to balance the weights of classification loss and sparsity loss.

\section{Discussion}

In this section, we verify the necessity of STGR in an analytical way. We first introduce an intuitive definition on "receptive field" and then point out that "star-structure" of human skeleton makes it hard for feature sharing between two limb nodes.

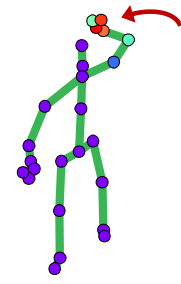

(a)

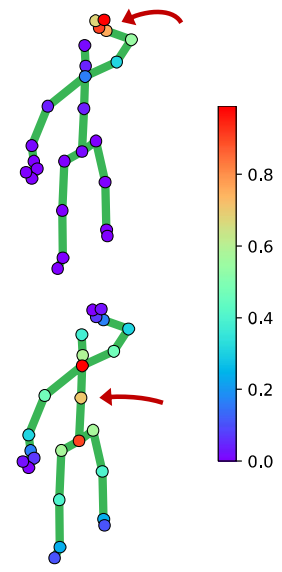

(b)

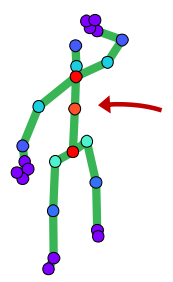

Figure 6: Comparison of the receptive of torso joint (lower back) and non-torso joint (right hand). (a) Receptive field of joint "right hand", left: after 3 steps diffusion. right: after 8 steps diffusion; (b) Receptive field of joint "lower back". left: after 3 steps diffusion. right: after 8 steps diffusion. Red color denotes high probability, purple color denotes low probability.

Receptive field is an important notation in CNNs which reveals the spatial context for a single neuron. By analogy with this idea, we introduce the concept "receptive field on graph", refering to the coverage range where a single node could draw information.

Figure 5 illustrates 3 types of connection mode. (a) denotes the predefined human skeleton while (b) and (c) show the learned connections with our SGR and TGR respectively. It is straightforward that the predefined skeleton organizes itself to form a "star-structure", in which a torso connects head and all four limbs. In this way, the centeral torso joints would spread far quicker than marginal limb joints, leading to great imbalance.

For illustration, we check the receptive field of a limb joint(right hand) and a torso joint(lower back) in Figure 6. Following previous literature(Xu et al. 2018), we cast the spreation of the Graph convolution into a $k$-step random walk process. The color represents the proportion of information which a node receive. As shown in Figure 6, after 3 steps diffusion, both two joints receive information from a relatively small range. After 8 steps the torso joint can nearly receive global information while the right hand joint still struggling in a small region.

Our proposed STGR scheme, from another point of view, learns pairwise connections from either postion or motion of joints, which breaks the above limitation. As depicted in Figure 5, the SGR learned graph mainly focuses on local gathering, in which close joints have strong connections. On the other hand, the TGR learned graph mainly put more attention to correlated joints in a long term. In this way, our STGR scheme effectively enlarges the receptive field for each joint and further promotes the trianing process. 


\section{Experiments}

In this section we evaluate our STGR scheme in skeletonbased action recognition datasets. We conduct experiments on two large-scale datasets: NTU-RGB+D and Kinetics. We first introduce our implementation details and then perform an ablation study on various settings of the spatio-temporal graph routing scheme. Last we compare our full model with other state-of-the-art approaches. All experiments are conducted on 4 GTX 1080Ti GPUs.

\section{Datasets}

NTU-RGB+D. NTU-RGB+D (Shahroudy et al. 2016) is a widely used large scale skeleton-based human action recognition dataset. It contains 56880 skeleton sequences with 60 action classes. The overall action classes are roughly divided as daily action, medical condition, and mutual action. Each action is captured by cameras at the same height but from three different horizontal angles: $-45^{\circ}, 0^{\circ}$, and $45^{\circ}$. Each human skeleton is represented as 3D-coordinates of 25 joints. Mutual action classes contain two subjects while the others contain only one subject. NTU-RGB+D recommends two evaluation protocals: 1). Cross-subject (X-Sub): The training and testing sets are divided into 40320 clips and 16560 clips respectively according to the difference of experiment subjects. 2). Cross-view (X-View): The training set is collected with camera view 2 and 3 with 37920 clips, while the evaluation set is collected from camera view 1 with 18960 clips. By following the convention of existing works (Yan, Xiong, and Lin 2018) for skeleton-based action recogntion, we report the top-1 accuracy on both two protocals.

Kinetics. Deepmind Kinetics is recently one of the largest human action dataset. The dataset contains nearly 300,000 video clips lasts around 10 seconds. To cover as many real occasions as possible, Kinetics collects videos from YouTube, composing 400 action classes. Note that raw Kinetics dataset contains only raw video clips. Following the previous practice (Yan, Xiong, and Lin 2018), we first extract raw 2D-coordinates with the help of OpenPose toolbox (Cao et al. 2017), then apply our model. Similar to NTU$\mathrm{RGB}+\mathrm{D}$, we extract 18 human joints and select 2 people with highest average joint confidence as main subject. In practice, we use the released data from (Yan, Xiong, and Lin 2018 ) to evaluate our model. The dataset is divided as training set with 240,000 clips and testing set with 20,000 clips. In this experiment, we report both top- 1 and top-5 accuracy.

\section{Implementation Details}

To ease computational burden, different from previous zero padding (Yan, Xiong, and Lin 2018), we first downsample sequence length to a fixed size 64 frames, which we conduct uniform sampling with bilinear interpolation when sequence length is larger than 64 while zero padding when the given sequence is shorter than 64 . In practice, we also conduct normalization for each frame, which makes training process more stable.

As for training, the whole network is trained with SGD optimizer with learning rate 0.1 for ST-GCN and 0.01 for

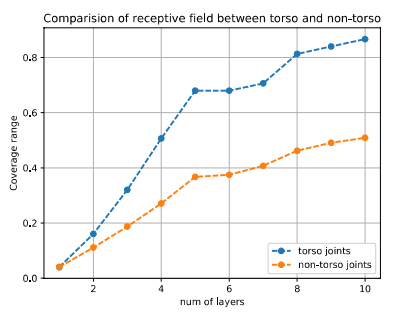

(a)

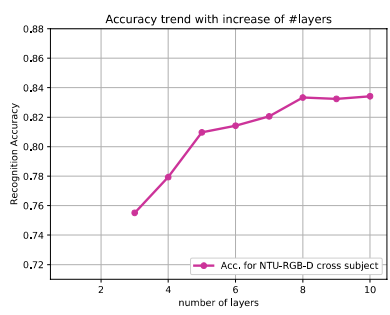

(b)
Figure 7: (a) Comparison of receptive field trends for torso joints and non-torso joints with increase of number of layers; (b) Recognition accuracy trend with increase of number of layers.

STGR. The weight decay is $1 \mathrm{e}-4$ and the batch size is set as 32. The balance parameter $\lambda$ of classification loss and $L 1$ loss is set as 0.2 since we mainly focus on classification result. We divide learning rate by 10 for both modules when monitoring validation loss stoping decrease over 5 epoches. Inspired by recent success ( $\mathrm{Li}$ et al. 2018a) on skeltetonbased action recognition, a "two-stream" scheme is applied to fuse both skeleton feature and motion feature. We need 60 training epoches for model convergence.

\section{Ablation Study}

In this section, we examine the effectivenss of our proposed STGR method and conduct experiments to test various parameter settings. All experiments are conducted on X-Sub benchmark on NTU-RGB+D dataset.

Receptive field In this work, we examine the necessity of STGR. According to Figure 7(a), we first divide all 25 joints into torso joints (root, lower back, upper back) and non-torso joints (the others). Then we calculate the average receptive field from 1 layer (1-step forward) to 10 layers (which we use in model) for two set respectively. The figure shows both two sets enlarge their receptive field with more layers. However, torso joints increase with an obvious faster speed, which verifies our analysis on human skeleton structure.

Additionally, we test the vanilla ST-GCN with different number of layers. We find the recognition accuracy follows the same trend with average receptive field. As shown in Figure 7(b), The accuracy increases fast initially. After 5 layers, the increasing speed becomes slow down. When approaching to 10 layers, the accuracy becomes stable. Stacking more layers would not affect the overall accuracy.

When reaching certain stage, both torso and limb joints are restricted into a relatively fixed range, which limits further improvement. It would be beneficial to very deep structure. However, such model brings large computational burden. In contrast, STGR effectively solves this problem by directly learning joint-joint connections.

Number of Groups As discussed before, in SGR, we first cluster skeleton in each frame into $K$ sub-groups. This procedure mines the implicit prior by directly exploring the relative distance. In practice, the cluster number $K$ is set as 5 by experiments. The results are shown in Table 1. 


\begin{tabular}{c|c}
\hline \# Clusters $K$ & Accuracy (\%) \\
\hline 3 & 84.63 \\
4 & 84.79 \\
5 & $\mathbf{8 5 . 2 2}$ \\
6 & 85.02 \\
7 & 84.84 \\
8 & 84.01 \\
\hline
\end{tabular}

Table 1: Comparison of the accuracies for different number of clusters.

In theory, too many clusters usually lead to over-splitting while under-splitting is mainly caused by too few clusters. We found that the performance is generally robust with its value ranging from 3 to 7 . Therefore, our choice just keeps a good balance.

Spatio-Temporal Graph Router As introduced above, the STGR is composed by two sub-networks - SGR and TGR. Table 2 shows that our proposed STGR can benefit for the vanilla ST-GCN.

\begin{tabular}{c|c}
\hline Methods & Accuracy (\%) \\
\hline Baseline (ST-GCN) & 83.38 \\
\hline STGR-GCN (w/SGR) & 85.22 \\
STGR-GCN (w/TGR) & 84.70 \\
STGR-GCN (full) & 85.80 \\
2s-STGR-GCN (full) & $\mathbf{8 6 . 9 8}$ \\
\hline
\end{tabular}

Table 2: Recognition accuracies with STGR module in NTU-RGB+D X-Sub.

In this part, we examine four variants of STGR-GCN to test the effectiveness of STGR module. The four variants includes: 1) GCN with spatial graph router; 2) GCN with temporal graph router; 3) GCN with spatio-temporal graph router; 4) Two stream GCN with spatio-temporal graph router.

The proposed method improves the baseline accuracy by $1.84 \%$ and $1.32 \%$ for SGR and TGR respectively. From the results, SGR performs slightly better than TGR. With the "two stream trick", the proposed model further improves by $1.18 \%$, which is an effective practice in skeleton-based action recognition.

\section{Comparison with state-of-the-art}

In this section, we evaluate our full STGR-GCN model with existed state-of-the-art skeleton-based action recognition models in NTU-RGB+D and Kinetics dataset.

NTU-RGB+D We roughly divide previous state-of-theart methods into four categories: 1) Hand-crafted methods: Lie group (Vemulapalli, Arrate, and Chellappa 2014); 2) RNN-based methods: STA-LSTM (Song et al. 2017), RNN-T/ACH (Li et al. 2017), GCA-LSTM (Liu et al. 2017); 3) CNN-based methods: Joint Trajectory Maps(Wang et al. 2018a), Skepxels (Liu, Akhtar, and Mian 2017), Temporal Conv (Kim and Reiter 2017), HCN (Li et al. 2018a); 4) Graph-based method: ST-GCN (Yan, Xiong, and Lin 2018).

\begin{tabular}{c|cc}
\hline \multirow{2}{*}{ Methods } & \multicolumn{2}{|c}{ Accuracy (\%) } \\
& X-Sub & X-View \\
\hline Lie group & 50.1 & 52.8 \\
\hline STA-LSTM & 73.4 & 81.2 \\
RNN-T/ACH & 74.6 & 83.2 \\
GCA-LSTM & 74.4 & 82.8 \\
\hline Joint Trajectory Maps & 76.3 & 81.1 \\
Temporal Conv & 74.3 & 84.1 \\
Skepxels & 81.3 & 89.2 \\
HCN & 86.5 & 91.1 \\
\hline ST-GCN & 81.5 & 88.3 \\
\hline STGR-GCN & $\mathbf{8 6 . 9}$ & $\mathbf{9 2 . 3}$ \\
\hline
\end{tabular}

Table 3: Recognition performance on NTU-RGB+D dataset. We compare out model with previous state-of-the-art on both crsss-subject(X-Sub) and cross-view(X-View).

Our STGR-GCN model, with simple spatio-temporal routing method, presents better results compared with vanilla ST-GCN and further achieves state-of-the-art, implying the effectiveness of dynamic routing scheme among graph convolution layers.

Kinetics On Kinetics, we compare our mdoel with one hand crafted approach: Feature encoding (Fernando et al. 2015); one RNN method: Deep LSTM (Shahroudy et al. 2016); a temporal-based CNN method: (Kim and Reiter 2017) and ST-GCN (Yan, Xiong, and Lin 2018). Following routine, we report both Top-1 and Top-5 accuracy.

\begin{tabular}{c|cc}
\hline \multirow{2}{*}{ Methods } & \multicolumn{2}{|c}{ Accuracy (\%) } \\
& Top-1 & Top-5 \\
\hline Feature Enc. & 14.9 & 25.8 \\
Deep LSTM & 16.4 & 35.3 \\
Temporal Conv & 20.3 & 40.0 \\
ST-GCN & 30.7 & 52.8 \\
\hline STGR-GCN & $\mathbf{3 3 . 6}$ & $\mathbf{5 6 . 1}$ \\
\hline
\end{tabular}

Table 4: Recognition performance on Kinetics dataset. We report Top- 1 and Top-5 accuracy.

\section{Conclusion}

This paper presents a novel routing scheme to generate spatio-temporal related graph for physically apart joints in skeleton-based action recognition, which solves the weakness of predefined human structure. Furthermore, we show the importance of constructing necessary connections by introducing receptive field on graph, which is effectively enlarged by our work. Qualitative and quantitative results are presented to verify the effectiveness of our method.

\section{Acknowledgements}

This work is supported in part by the National Natural Science Foundation of China under Grants (61672456, U1509206, 61472353, and 61751209), Zhejiang Provincial Natural Science Foundation of China under Grant 
LR19F020004, ZhiJiang Lab (2018EC0ZX01-2), the National Basic Research Program of China under Grant 2015CB352302, Zhejiang University K.P.Chao's High Technology Development Foundation, the fundamental research funds for central universities in China (2017FZA5007), Zhejiang provincial engineering research center on network media data cloud processing and analysis technologies, Tencent AI Lab Rhino-Bird Joint Research Program(No. JR201806), and the funding from HIKVision and ZJU Converging Media Computing Lab.

\section{References}

Bruna, J.; Zaremba, W.; Szlam, A.; and Lecun, Y. 2014. Spectral networks and locally connected networks on graphs. In International Conference on Learning Representations (ICLR2014), CBLS, April 2014.

Cao, Z.; Simon, T.; Wei, S. E.; and Sheikh, Y. 2017. Realtime multi-person $2 \mathrm{~d}$ pose estimation using part affinity fields. In IEEE Conference on Computer Vision and Pattern Recognition, 1302-1310.

Defferrard, M.; Bresson, X.; and Vandergheynst, P. 2016. Convolutional neural networks on graphs with fast localized spectral filtering. In Advances in Neural Information Processing Systems, 3844-3852.

Fernando, B.; Gavves, E.; Oramas, M. J.; Ghodrati, A.; and Tuytelaars, T. 2015. Modeling video evolution for action recognition. In Computer Vision and Pattern Recognition, 5378-5387.

Hammond, D. K.; Vandergheynst, P.; and Gribonval, R. 2011. Wavelets on graphs via spectral graph theory. Applied and Computational Harmonic Analysis 30(2):129-150.

Hu, J.; Shen, L.; and Sun, G. 2018. Squeeze-and-excitation networks. In The IEEE Conference on Computer Vision and Pattern Recognition (CVPR).

Hussein, M. E.; Torki, M.; Gowayyed, M. A.; and El-Saban, M. 2013. Human action recognition using a temporal hierarchy of covariance descriptors on $3 \mathrm{~d}$ joint locations. In IJCAI, volume 13, 2466-2472.

Kay, W.; Carreira, J.; Simonyan, K.; Zhang, B.; Hillier, C.; Vijayanarasimhan, S.; Viola, F.; Green, T.; Back, T.; and Natsev, P. 2017. The kinetics human action video dataset.

Kim, T. S., and Reiter, A. 2017. Interpretable 3d human action analysis with temporal convolutional networks. In IEEE Conference on Computer Vision and Pattern Recognition Workshops, 1623-1631.

Kipf, T. N., and Welling, M. 2017. Semi-supervised classification with graph convolutional networks. In International Conference on Learning Representations (ICLR).

Li, W.; Wen, L.; Chang, M. C.; Lim, S. N.; and Lyu, S. 2017. Adaptive rnn tree for large-scale human action recognition. In IEEE International Conference on Computer Vision, 1453-1461.

Li, C.; Zhong, Q.; Xie, D.; and Pu, S. 2018a. Co-occurrence feature learning from skeleton data for action recognition and detection with hierarchical aggregation. In IJCAI, 786792.
Li, R.; Wang, S.; Zhu, F.; and Huang, J. 2018b. Adaptive graph convolutional neural networks. arXiv preprint arXiv:1801.03226.

Liu, J.; Akhtar, N.; and Mian, A. 2017. Skepxels: Spatiotemporal image representation of human skeleton joints for action recognition. arXiv preprint arXiv:1711.05941.

Liu, J.; Wang, G.; Hu, P.; Duan, L.-Y.; and Kot, A. C. 2017. Global context-aware attention lstm networks for 3d action recognition. In The IEEE Conference on Computer Vision and Pattern Recognition (CVPR), volume 7, 43.

Niepert, M.; Ahmed, M.; and Kutzkov, K. 2016. Learning convolutional neural networks for graphs. In International conference on machine learning, 2014-2023.

Shahroudy, A.; Liu, J.; Ng, T.-T.; and Wang, G. 2016. Ntu $\mathrm{rgb}+\mathrm{d}$ : A large scale dataset for $3 \mathrm{~d}$ human activity analysis. In Proceedings of the IEEE conference on computer vision and pattern recognition, 1010-1019.

Shi, J., and Malik, J. 2000. "normalized cuts and image segmentation", ieee trans. IEEE Trans.pattern Anal.mach.intell 22(8):888-905.

Shuman, D. I.; Narang, S. K.; Frossard, P.; Ortega, A.; and Vandergheynst, P. 2013. The emerging field of signal processing on graphs: Extending high-dimensional data analysis to networks and other irregular domains. IEEE Signal Processing Magazine 30(3):83-98.

Song, S.; Lan, C.; Xing, J.; Zeng, W.; and Liu, J. 2017. An end-to-end spatio-temporal attention model for human action recognition from skeleton data. In $A A A I$, volume 1 , 4263-4270.

Vemulapalli, R.; Arrate, F.; and Chellappa, R. 2014. Human action recognition by representing $3 \mathrm{~d}$ skeletons as points in a lie group. In IEEE Conference on Computer Vision and Pattern Recognition, 588-595.

Wang, P.; Li, W.; Li, C.; and Hou, Y. 2018a. Action recognition based on joint trajectory maps with convolutional neural networks. Knowledge-Based Systems.

Wang, P.; Li, W.; Ogunbona, P.; Wan, J.; and Escalera, S. 2018b. Rgb-d-based human motion recognition with deep learning: A survey. Computer Vision and Image Understanding.

Wang, X.; Girshick, R.; Gupta, A.; and He, K. 2018c. Nonlocal neural networks. In The IEEE Conference on Computer Vision and Pattern Recognition (CVPR).

Xie, C.; Li, C.; Zhang, B.; Chen, C.; Han, J.; and Liu, J. 2018. Memory attention networks for skeleton-based action recognition. In IJCAI, 1639-1645.

Xu, K.; Li, C.; Tian, Y.; Sonobe, T.; Kawarabayashi, K.i.; and Jegelka, S. 2018. Representation learning on graphs with jumping knowledge networks. arXiv preprint arXiv:1806.03536.

Yan, S.; Xiong, Y.; and Lin, D. 2018. Spatial temporal graph convolutional networks for skeleton-based action recognition. In $A A A I$. 